\section{Pas de santé sans santé mentale}

La santé mentale est une composante essentielle de la santé. Lors de la Journée mondiale de la santé mentale, célébrée le 10 octobre, il est important de rappeler la Constitution de l'OMS qui définit la santé comme étant «un état de complet bien-être physique, mental et social». Les politiques nationales de santé mentale ne doivent pas limiter leur champ d'action aux troubles mentaux. Il faut aussi qu'elles reconnaissent et prennent en compte les facteurs plus généraux qui favorisent la santé mentale. Il s'agit notamment d'intégrer la promotion de la santé mentale dans les politiques et programmes des secteurs publics et non gouvernemental.

\section{Studie «nurses at work»}

Die nationale Studie «nurses at work» untersucht die Hintergründe, weshalb genau diplomierte Pflegefachpersonen im Beruf bleiben - oder diesen verlassen. Die Studie will in Erfahrung bringen, welche konkreten Antworten im Kampf gegen den Personalmangel im Pflegebereich zu finden sind. Aufgrund der Erkenntnisse sollen konkrete und umsetzungsorientierte Vorschläge zur Erhöhung der Berufsverweildauer von Pflegefachpersonen abgeleitet werden. Curaviva Schweiz unterstützt zusammen mit einem grossen Netzwerk von Partnern das Forschungsteam beim Aufruf zur Studienbeteiligung von möglichst vielen aktiven oder ehemaligen diplomierten Pflegefachpersonen.

(Curaviva)
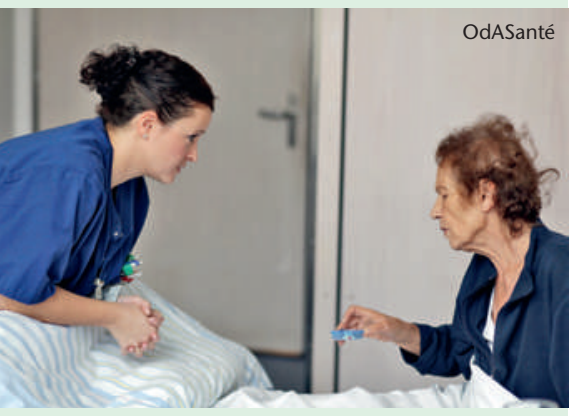

Warum verlassen Pflegefachpersonen ihr Berufsfeld?

\section{«Sieh dich vor beim Schritt auf die Strasse»}

«Ein Augenblick der Unaufmerksamkeit kann dein Leben für immer verändern»: Das ist der Kern der Verkehrssicherheitskampagne von Fussverkehr Schweiz, die Ende September lanciert wurde. Der Kampagnenfilm, den Fussverkehr Schweiz in Zusammenarbeit mit dem Ballett Zürich realisiert hat, zeigt: Ein verträumter Gang durch den Tag kann abrupt in der Katastrophe enden. Im ersten Halbjahr 2014 verloren auf Schweizer Strassen 15 Fussgänger ihr Leben. Nur drei von ihnen verunglückten auf einem Fussgängerstreifen. Das Queren der Fahrbahn ohne Fussgängerstreifen ist somit Schwerpunkt des Unfallgeschehens. Eine Auswertung zeigt, dass Unfälle beim Queren an einem Ort ohne Fussgängerstreifen mit schwereren Verletzungen der Opfer verbunden sind.

(Fussverkehr Schweiz)

\section{Pilzgenuss ohne Reue}

Speisepilze selber zu sammeln, macht Spass. Aber bekanntlich sind nicht alle Pilze geniessbar. Die Verwechslung giftiger Doppelgänger essbarer Speisepilze ist die grösste Gefahr. Das Bundesamt für Lebensmittelsicherheit und Veterinärwesen BLV rät zur Vorsicht und ruft alle Sammler auf, ihr Sammelgut kontrollieren zu lassen. Gesammelte Pilze, die selber verzehrt werden, fallen nicht unter die Lebensmittelgesetzgebung (Eigengebrauch). Beim
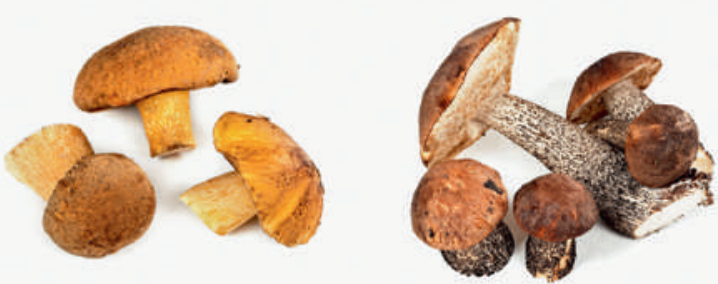

\section{Ambizione: subsides distribués à 60 jeunes chercheurs}

Cette année, le Fonds Nationale Suisse FNS a alloué 60 subsides à la relève scientifique suite à la mise au concours des instruments Ambizione, Ambizione-PROSPER et Ambizione-SCORE. A la mifévrier 2014, 294 jeunes chercheuses et chercheurs avaient déposé une requête dans le cadre d'Ambizione. Après un processus de sélection en deux étapes, le FNS a attribué au total 60 nouveaux subsides. Avec 19 subsides octroyés à des femmes (soit un peu moins de $32 \%$ ), l'objectif des 35\% de bénéficiaires féminines est presque atteint cette année. Par le biais d'Ambizione, Ambizione-PROSPER et Ambizione-SCORE, le FNS permet à la relève scientifique de faire ses premiers pas dans la recherche indépendante, et ce dans toutes les disciplines. La prochaine mise au concours de l'instrument Ambizione est prévue dès la mi-novembre 2014

(FNS)
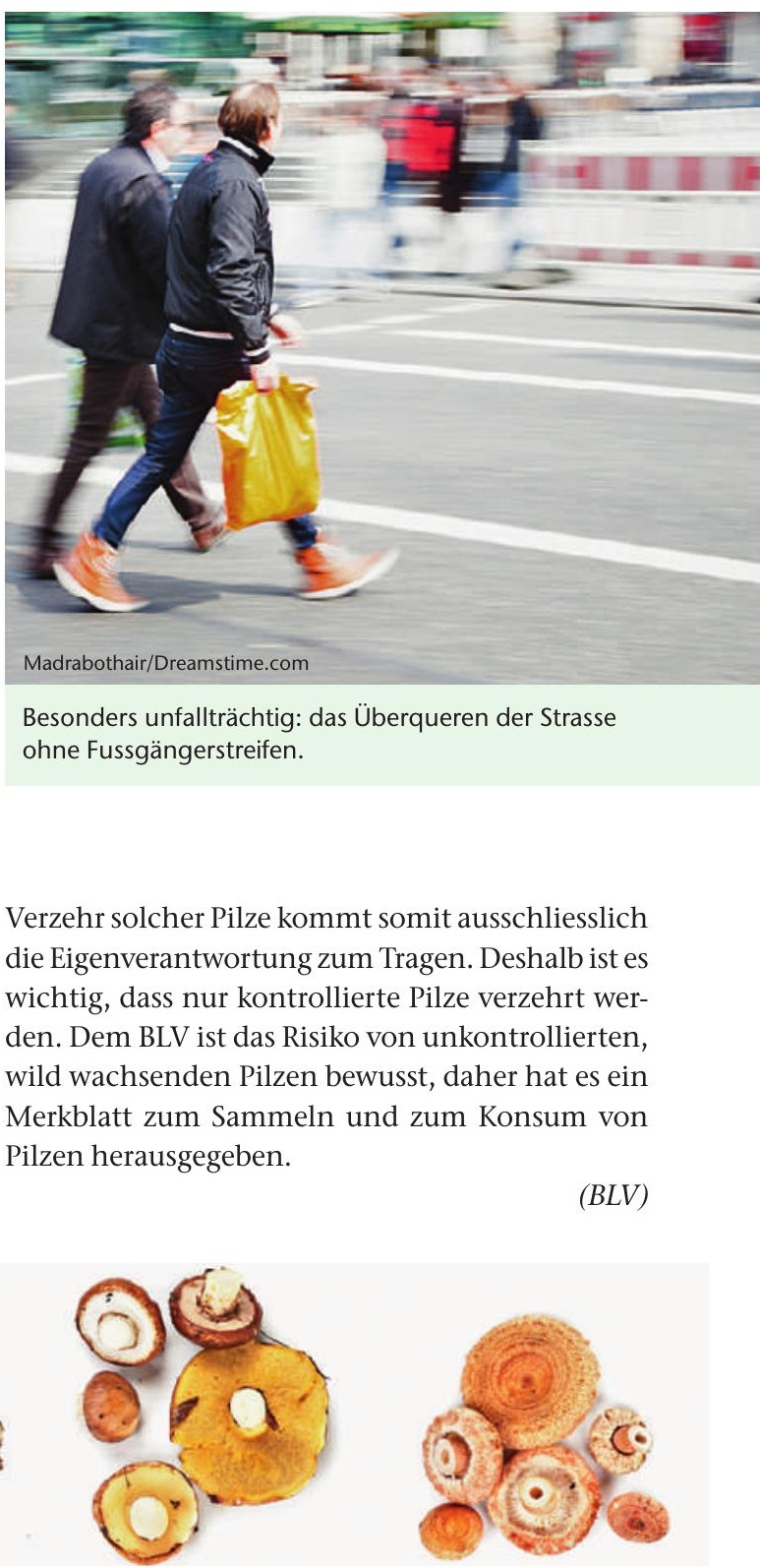

Besonders unfallträchtig: das Überqueren der Strasse ohne Fussgängerstreifen.

Verzehr solcher Pilze kommt somit ausschliesslich die Eigenverantwortung zum Tragen. Deshalb ist es wichtig, dass nur kontrollierte Pilze verzehrt werden. Dem BLV ist das Risiko von unkontrollierten, wild wachsenden Pilzen bewusst, daher hat es ein Merkblatt zum Sammeln und zum Konsum von Pilzen herausgegeben.

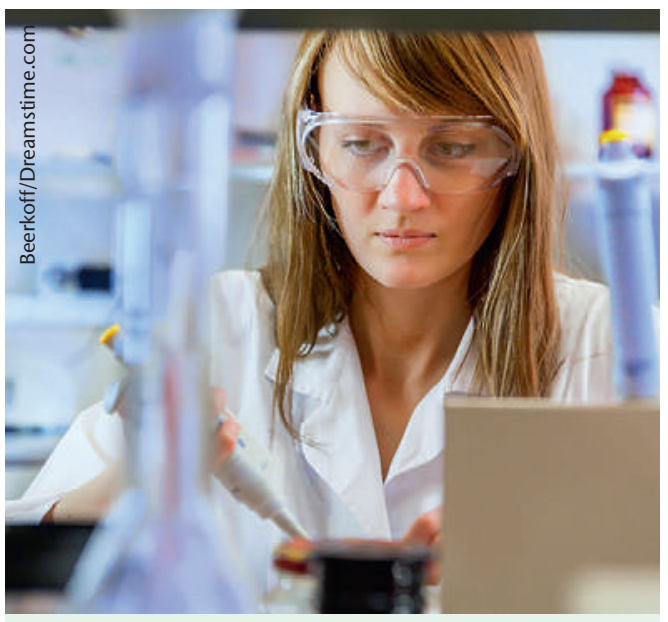

L'objectif des $35 \%$ de bénéficiaires féminines est presque atteint cette année. 